\title{
RHOMBENCEPHALITIS: A RARE MANIFESTATION OF SYSTEMIC LUPUS ERYTHEMATOSUS ACTIVITY
}

Evelise Mileski do Amaral Berlet ${ }^{1, \star}$, Marcel Mathias Villaça ${ }^{1}$, Júlia Boechat Farani ${ }^{1}$, Erika Biegelmeyer ${ }^{1}$, Marília Voges de Souza ${ }^{1}$, Samanta Gerhardt ${ }^{1}$, Cristina Ferreira Rabelo ${ }^{1}$

1.Pontifícia Universidade Católica do Rio Grande do Sul, Porto Alegre (RS), Brazil.

*Corresponding author: liseamaral@hotmail.com

\section{BACKGROUND}

Rhombencephalitis - inflammation of the brainstem and cerebellum — has a myriad of clinical presentations and a variety of potential underlying causes including infections, inflammatory disorders, autoimmune encephalitis and paraneoplastic syndromes.

\section{CASE REPORT}

A 24-year-old Caucasian woman, with previous diagnosis of systemic lupus erythematosus (SLE) and irregular treatment was admitted to our hospital with a 4-week history of fever, headache, visual disturbance and diffuse cutaneous vasculitic lesions. Physical examination evidenced meningism and symmetrical proximal weakness of lower limbs. Magnetic resonance imaging (MRI) suggested an inflammatory process involving pons, bulb and left midbrain. Furthermore, leptomeningeal signal enhancement of the cerebellar folia and the left occipital lobe was evidenced. These findings suggested the occurrence of ongoing rhombencephalitis. Cerebrospinal fluid (CSF) analysis showed albumin cytologic dissociation and negative microbiological workup, including negative polymerase chain reaction for Listeria monocytogenes. Other laboratorial tests showed complement consumption, reagent anti-DNA antibody, pyuria and high levels of urinary casts. Because of the uncertain diagnosis at the admission, she received empirical intravenous (IV) antibiotic therapy (ampicillin) and IV methylprednisolone infusion in a total dose of 5,000 mg for 21 days, with progressive clinical and laboratorial improvement. She developed SARS-CoV-2 pneumonia during hospitalization, which contraindicated pulse therapy with cyclophosphamide or rituximab. A control MRI performed after 21 days of treatment demonstrated regression of the findings of rhombencephalitis, and new CSF collection showed absence of protein content of the cerebrospinal fluid. After 6-weeks, the patient was discharged from the hospital without neurological symptoms, with complete recovery from the proximal weakness of the lower limbs.

\section{CONCLUSION}

Rhombencephalitis is an uncommon neurological condition with potentially devastating sequelae. Infection conditions (viral or bacterial) are the most frequent cause of this disorder, mainly infection with the Gram-positive bacteria Listeria monocytogenes. Although listeriosis can affect the immunocompetent, it typically presents in the elderly, immunocompromised or pregnant population, manifesting as meningitis or meningoencephalitis. The risk seems particularly higher among users of infliximab (an antitumor necrosis factor-a medication). Rhombencephalitis develops in about $9 \%$ of patients with listeria infections of the central nervous system. Among the autoimmune conditions, the most common to develop brainstem disease is the neuro-Behçet' syndrome. On the other hand, SLE is rarely associated with rhombencephalitis. Therefore, a complete assessment of the clinical history and general plus neurological physical examination are crucial to guide prompt and appropriate investigation and treatment.

\section{KEYWORDS}

Rhombencephalitis, Systemic lupus erythematosus, Autoimmune encephalitis. 